\title{
Unidos somos más fuertes. Cooperación en las bibliotecas científicas alemanas ${ }^{1}$
}

\author{
Por Klaus Kempf
}

\begin{abstract}
Resumen: La cooperación bibliotecaria es en Alemania una tradición anterior a la aparición de internet. Esto se explica, por un lado, por la ausencia de una Biblioteca Nacional "clásica", entendida como una única institución central. Esta ausencia ha sido suplida por las tres mayores bibliotecas alemanas, que se han distribuido la función propia de una biblioteca nacional. Por otro lado, el sistema político federal y la responsabilidad de cada uno de los estados federales en los asuntos culturales, entre ellos las bibliotecas, ha originado que, tanto en el plano regional como interregional, haya habido tradicionalmente una sólida cooperación, al menos entre las bibliotecas científicas. Posteriormente, la llegada de la automatización ha hecho posible la aparición de los catálogos colectivos regionales. Con la entrada en la llamada "era digital", se ha intensificado aún más la tendencia a la cooperación, de modo que hoy día se puede hablar de una "era de la cooperación". Muestra de la mayor facilidad y agilidad de la misma son los nuevos servicios, que en muchos casos suministran conjuntamente varias bibliotecas, como las llamadas bibliotecas virtuales especializadas, la adquisición consorciada de licencias de uso de medios digitales, entre las que se incluyen las licencias nacionales, y la creación cooperativa de un archivo digital nacional de larga preservación.
\end{abstract}

Palabras clave: Cooperación bibliotecaria, Bibliotecas científicas alemanas, Bibliotecas virtuales, Era digital.

\section{Title: United we are stronger. Cooperation among the German scientific libraries}

Abstract: Library cooperation in Germany is a tradition that predates the emergence of internet. This is explained partly by the absence of a "classic" National Library, as a single central institution. This absence has been replaced by the three major German libraries, which have distributed among themselves the function of a national library. The other reason for this tradition is the Federal political system and the responsibility of each of the Federal States in cultural matters, including libraries. This meant that at both regional and interregional levels, there has traditionally been a solid cooperation, at least among scientific libraries. Later, the arrival of automation has facilitated the production of collective regional catalogues. With the entrance into the so-called "digital age", the tendency to cooperation has been intensified, so that today one can talk about an "age of cooperation". One example of the ease and assiduousness of this cooperation are the new services which, in most cases, supply several libraries together; others are the specialised virtual libraries, the consortium acquisition of digital media user licences, including national licences for certain items, and the cooperative creation of a long term national digital archive.

Keywords: Library cooperation, German scientific libraries, virtual libraries, digital age.

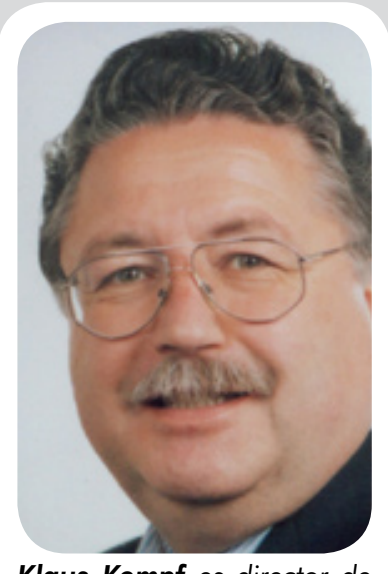

Klaus Kempf es director de la Sección de Desarrollo de la Colección y Catalogación de la Bayerische Staatsbibliothek y responsable de la puesta en funcionamiento del Centro de digitalización (uno de los dos centros de digitalización nacional en Alemania). Desde el año 2000 es coordinador jefe del Consorcio de Baviera y miembro desde su fundación de Gasco (Grupo de trabajo de los consorcios regionales de habla alemana, incluidos Austria y Suiza). Es autor de numerosas publicaciones en revistas especializadas de biblioteconomía, alemanas, italianas e internacionales, especialmente sobre temas relacionados con la organización, la logística, las adquisiciones y la gestión, así como sobre cuestiones arquitectónicas y de instalaciones

Kempf, Klaus. "Unidos somos más fuertes. Cooperación en las bibliotecas científicas alemanas”. En: El profesional de la información, 2008, mayo-junio, vol. 17, n. 3, pp. 324-330.

DOI: 10.3145/epi.2008.may.09

\section{Introducción}

EL CONCEPTO DE DIVISIÓN DEL TRABAJO y de networking está hoy día en boca de todos. El ámbito bibliotecario no es una excepción. Por el contrario, al menos en el mundo bibliotecario científico en Alemania, no es una moda reciente, sino una forma de trabajar desde hace décadas, una verdadera tradición.

Naturalmente, los contenidos y modos de la cooperación han ido cambiando con el tiempo, pero el objetivo ha sido siempre el mismo: ofrecer al usuario de modo conjunto o mediante una distribución de tareas un servicio que:
- le proporcione la mayor utilidad;

- implique una reducción de costes para la biblioteca o las bibliotecas participantes.

\section{Aclaración del concepto}

Por lo que respecta a la cooperación bibliotecaria es válido, en 
gran parte, lo que se entiende por cooperación cuando se lleva a cabo entre empresas. Según el OnlineBrockhaus, que es la enciclopedia alemana más conocida, es definida como el trabajo conjunto -por lo general regulado mediante contrato-, que empresas tanto jurídica como económicamente independientes llevan a cabo de modo voluntario para mejorar su rendimiento. Puede dar lugar a la creación de compañías comunes para proyectos compartidos.

Se pueden diferenciar así diversos tipos de cooperación:

- Horizontal (empresas de la misma área económica).

- Vertical (con tareas diferentes).

- Mixta (empresas de tareas y ámbitos económicos diversos).

De acuerdo con esto, las organizaciones cooperantes pueden presentarse externamente como comunidad de intereses, consorcio, alianza estratégica, etc.

Todas las formas de cooperación están hoy presentes en el mundo bibliotecario. En lo que sigue, quisiera ocuparme sobre todo de aquella de tipo horizontal, es decir, la que se realiza entre bibliotecas -más exactamente las de tipo científico en Alemania- centrándome principalmente en mi propio Estado federal: Baviera² .

\section{Cooperación tradicional en las bibliotecas científicas alemanas}

Estos centros se han construido, como ya se ha dicho, sobre la base de la cooperación. Esto es así tanto en el plano regional, interregional y nacional, como en lo que respecta a las diversas tareas bibliotecarias: adquisición, catalogación, circulación y uso de la colección, aunque con diferente intensidad y amplitud. Resulta necesario ofrecer algunas indicaciones sobre el marco político general en el que se mueven las bibliotecas alemanas, porque sólo así se puede entender adecuadamente el concepto de cooperación.

\subsection{Marco político general}

El mundo bibliotecario alemán tiene como principales características las siguientes:

- Prácticamente todas las bibliotecas son de titularidad pública, esto es, están gestionadas por el Estado Central, los Estados federales o los municipios y, por consiguiente, forman parte del sector de los servicios públicos.

- Según la Constitución de la República Federal de Alemania, cada Estado federal es el responsable principal de los asuntos de orden cultural y educativo, que incluyen todo lo relacionado con las bibliotecas científicas. El Estado Central interviene sólo de modo secundario. Ciertamente, toma parte en las decisiones acerca del desarrollo de las instituciones de educación superior que afectan, por ejemplo, a la financiación de la dotación informática y de la construcción de las bibliotecas, e interviene indirectamente en otros asuntos esenciales para las mismas. Hay que mencionar además el fomento por parte del Estado Central de la investigación fuera de la universidad, que en Alemania tiene gran importancia y que se lleva a cabo por lo general a través de instituciones de ámbito estatal, ya sea por medio de proyectos financiados por la Deutsche Forschungsgesellschaft (Sociedad Alemana de Investigación) (véase más adelante), o incluso mediante organizaciones específicas, como la Max Planckgesellschaft, Leibnizgesellschaft o los Helmholtz-Institute, que disponen de magníficas bibliotecas especializadas.

- En el contexto de la autonomía administrativa, los municipios deciden sobre sus propias bibliotecas públicas. Para ello reciben ayuda financiera de su Estado federal en las convocatorias públicas para proyectos bibliotecarios.

\subsection{Biblioteca Nacional Alemana Virtual}

A nivel nacional es preciso mencionar un fenómeno cooperativo muy especial. Alemania nunca ha tenido una biblioteca nacional "clásica", siguiendo la definición de la Unesco o la IFLA, como Gran Bretaña, Francia o España, ni tampoco una biblioteca científica sobresaliente que desempeñara de hecho la función de una biblioteca nacional, como por ejemplo la Library of Congress en Estados Unidos, es decir, una única institución central. Estas funciones son llevadas a cabo de modo conjunto por tres bibliotecas, a saber:

\section{"Alemania nunca ha tenido una biblioteca nacional 'clásica', siguiendo la definición de la Unesco o la IFLA, como Gran Bretaña, Francia o España"}

- El centro bibliográfico nacional de la Biblioteca Alemana o, como se denomina oficialmente desde el 1 de junio de 2007, la $B i$ blioteca Nacional Alemana (DNB), con sede en Frankfurt, Leipzig y Berlín (en este último lugar tiene la sede el Archivo Musical Alemán, que figura como una sección de la $D N B$ ); reúne exclusivamente fondo bibliográfico en alemán y edita la Bibliografía Nacional Alemana.

- La Biblioteca Estatal de Berlín, es decir, la antigua Biblioteca Estatal Prusiana.

- La Biblioteca Estatal de Baviera.

Las dos últimas instituciones mencionadas son las dos mayores bibliotecas del ámbito lingüístico 
alemán y tradicionalmente, además de la literatura alemana, coleccionan sobre todo materiales en lenguas extranjeras, es decir, aquellos materiales que no recoge la $D N B$.

Ya antes de la aparición de internet se hablaba, por tanto, de una "biblioteca nacional alemana virtual", dado que tres instituciones situadas en lugares diferentes realizaban esas tareas de modo virtual y cooperativo.

Recientemente se ha ampliado el círculo de bibliotecas que se ocupan sistemáticamente de la tarea de reunir y conservar del modo más completo posible la bibliografía en lengua alemana. En el denominado Grupo de trabajo para la colección de impresos alemanes (SDD), se ha distribuido la tarea del modo siguiente:

- La Biblioteca Estatal de Baviera $(B S B)$, responsable de todo el fondo impreso alemán de 1450 a 1600 (además, es la única responsable de la adquisición de todo el material musical de los siglos XV a XVIII).

- La Herzog-August-Bibliothek, en Wolfenbuittel, del fondo impreso alemán desde 1601 a 1700.

- La Biblioteca Estatal y Universitaria de Baja Sajonia, en Gotinga, del fondo impreso alemán desde 1701 a 1800.

- La Biblioteca de la Universidad de Frankfurt, del fondo impreso alemán desde 1801 a 1870.

- La Biblioteca Estatal de Berlín, del fondo impreso alemán desde 1871 a 1912.

- La Biblioteca Nacional Alemana, por último, es responsable del fondo impreso alemán desde 1913 hasta el presente.

Todas ellas se comprometen a examinar de modo sistemático la totalidad de las ofertas existentes en el mercado de anticuarios para el correspondiente período de tiempo, y dado el caso, a adquirir aquellos títulos que no se encuentran en su fondo bibliográfico. Por esta razón las nuevas adquisiciones no se incluyen sólo en los propios catálogos locales, sino también -e igualmente de modo cooperativo- en bases de datos especiales, como VD 16, VD 17, el censo de incunables o $B S B-I N K$, así como los mapas en IKAR o en Kalliope (base de datos nacional de autógrafos: Nachlässe), administrada por la biblioteca de Berlín, y la Handschriftendatenbank (base de datos nacional de manuscritos antiguos). Finalmente, las publicaciones periódicas se incluyen en la base de datos nacional de revistas $(Z D B)$, que actualmente contiene casi 5 millones de títulos.

Hay que decir que todas las bases de datos antes comentadas están abiertas a todas las bibliotecas de Alemania y el trabajo de estas estructuras en red puede ser considerado como una verdadera cooperación a nivel nacional.

\subsection{Agrupaciones regionales de bibliotecas}

Otra tradicional división del trabajo en el mundo de las bibliotecas científicas alemanas tiene lugar en el nivel regional e interregional, a través de las llamadas Agrupaciones de bibliotecas. Son una consecuencia del desarrollo de los años 70 y de la introducción de la informática en este contexto. Con este motivo, y especialmente con la automatización de las tareas de catalogación, se consideró conveniente crear agrupaciones y centros coordinadores que se ocuparan de las cuestiones específicas de informática en las bibliotecas. Hay dos modelos: o bien la unión de bibliotecas coincide con el correspondiente Estado federal, de quien depende su mantenimiento (como por ejemplo en Baviera, donde la Biblioteca Central del Estado, en este caso la $B S B$, actúa como centro coordinador), o bien en los casos en que el
Estado federal era muy pequeño o disponía de muy pocas bibliotecas, la agrupación se organiza de modo suprarregional, con un centro coordinador común para varios Estados federales, como por ejemplo la Agrupación Conjunta de Bibliotecas $(G B V)$ de los Estados federales del norte de Alemania, cuyo centro coordinador es la Biblioteca Estatal y Universitaria de Baja Sajonia, Gotinga.

En la actualidad existen 6 agrupaciones de bibliotecas que disponen a nivel nacional de un organismo coordinador y trabajan conjuntamente. Es preciso señalar que sus funciones van mucho más allá de lo que supone encargarse de mantener los respectivos catálogos colectivos. Constituyen centros de servicios digitales multifuncionales, que ofrecen a sus respectivas bibliotecas asociadas múltiples servicios, entre los que el apoyo en la catalogación continúa siendo la tarea nuclear.

Los catálogos colectivos regionales o sus versiones opac son la base organizativa y técnica para un servicio destinado a las bibliotecas alemanas, que consiste en un sistema de préstamo nacional (interbibliotecario), que funciona aceptablemente y que está cada vez más automatizado, a partir de una petición online del usuario. La base logística, es decir, el transporte de los libros, la proporciona el Servicio de transporte de libros en Alemania $(B T D)$, iniciado hace 10 años, que llega a todas las regiones alemanas.

http://www.buechertransportdienst.de/

¿Cómo funciona este servicio tan importante para el usuario?

A partir de una búsqueda bibliográfica de una monografía, por ejemplo en el catálogo colectivo de Baviera (Gateway Bayern), que no produce resultado positivo en la biblioteca local, se ofrece al usuario como alternativa de modo automático -mediante un botón- el présta- 
mo interbibliotecario de la obra solicitada. En primer lugar, el servidor central de préstamo interbibliotecario busca mediante un determinado algoritmo en los catálogos de la propia región (en el caso mencionado, los fondos existentes en el $B V B$ ). Si no lo encuentra, la consulta se realiza mediante un nuevo proceso en el servidor central de préstamo interbibliotecario de las regiones vecinas. En caso de necesidad se consultan en tiempo real todos los centros de préstamo interbibliotecario de las agrupaciones regionales alemanas. Todo ello sin que sea visualizado por el usuario, al que le da igual que el libro solicitado esté en Hamburgo, Colonia o Berlín. Lo importante es que lo reciba rápidamente. Normalmente, podrá sacarlo en préstamo en 48 ó 72 horas en la biblioteca de Baviera en que lo solicitó, aunque el centro que lo presta esté situado en Renania Westfalia. La ventaja más importante es que el usuario recibe el libro en un tiempo relativamente breve y los costes para las bibliotecas participantes son muy reducidos.

En Baviera el préstamo interbibliotecario de libros es todavía gratuito para el usuario; en las demás regiones se paga una tasa fija reducida. El usuario, además, puede solicitar por el mismo procedimiento artículos de revistas. El envío es, por lo general, más rápido: por fax o en formato electrónico, mediante un documento adjunto de correo electrónico de la biblioteca prestataria a la del usuario solicitante. La automatización del préstamo interbibliotecario es un gran logro y los usuarios lo utilizan activamente. Una muestra de ello es la evolución de las solicitudes atendidas: de las 525.000 en 2003 se ha pasado a más de un millón en 2007.

\subsection{Red nacional de colecciones especiales (plan de colecciones te- máticas especiales de la $D F G$ )}

Un tercer ejemplo de la tradicionalmente fuerte división del trabajo en el mundo bibliotecario alemán lo constituye la red de las llamadas bibliotecas de colecciones temáticas especiales. Originalmente surgió como medida de emergencia de la Deutsche Forschungsgemeinschaft (Sociedad Alemana de Investigación) tras la Segunda Guerra Mundial. En la actualidad, el plan de colecciones, que abarca todas las áreas y temas científicos, así como todos los idiomas y países, incluye más de 70 bibliotecas. Lo esencial es que las bibliotecas implicadas, además de sus propias tareas en ocasiones muy diversas, se comprometen a reunir sistemáticamente toda la bibliografía mundial de determinados temas y/o países y lenguas. Para ello reciben financiación de la $D F G$ (que cubre el $75 \%$ del coste de la adquisición de la literatura extranjera y en otros idiomas). Este proyecto cooperativo refleja de modo especial, una vez más, las características histórico-políticas propias del ámbito bibliotecario alemán, es decir, por un lado, la ausencia de una instancia central encargada de la función de reunir una colección completa y, por otro, la estructura federal de la actual constitución política.

Las bibliotecas de colecciones temáticas especiales desempeñan hoy, de modo destacado, la función de centros digitales con una oferta de información propia y especializada por temas, como se verá más adelante. Además de las dos grandes bibliotecas generales en Berlín y en Múnich, se incluyen también las denominadas bibliotecas centrales especializadas por temas (medicina en Colonia, ciencias económicas en Kiel, ciencias técnicas e ingeniería en Hannover), así como una amplia relación de bibliotecas universitarias y especializadas. Todas ellas participan en el servicio de suministro de documentos (document delivery service) Subito, que es otro proyecto cooperativo, que también atiende solicitudes del extranjero y cuyos servicios segu- ramente son conocidos en España. El servicio ha asentado sobre nuevas bases organizativas y técnicas el suministro de información especializada para cualquier usuario en Alemania. A diferencia del servicio similar de la British Library, en Subito se encuentran a disposición de cada una de las bibliotecas participantes todos los fondos propios, en algunos casos muy especializados, en las mismas condiciones $y$, sobre todo, a un mismo precio. Esto no impide a las bibliotecas suministradoras -en la actualidad participan activamente como tal más de 30 proporcionar otros servicios como, por ejemplo, el portal BioMed de la Bayerische Staatsbibliothek. Por desgracia, la reciente reforma del derecho de propiedad intelectual en Alemania prohibe, desde el 1 de julio de 2007, el suministro por vía digital, o lo hace considerablemente más caro. Lamentamos mucho esta nueva situación, que muestra una vez más que las bibliotecas no encuentran suficiente apoyo para sus propios intereses y los de sus usuarios ni en Alemania ni en Europa, donde se toman o se preparan todas las decisiones políticas. El libre acceso continúa siendo un piadoso sueño.

\section{"La reciente reforma del derecho de propiedad intelectual en Alemania prohibe, desde el 1 de julio de 2007, el suministro por vía digital, o lo hace considerablemente más caro"}

\section{Cooperación en la era digital}

\subsection{Era digital = Era de la coope- ración}

Hasta ahora he tratado de mostrar, a partir de ejemplos concretos, 
los modos tan diversos de cooperación bibliotecaria en Alemania, tanto en el plano regional, como interregional y nacional. Con la aparición de internet y la llegada de la información digital, no quedaron obsoletas las condiciones marco características de Alemania: un país con estructura federal, en el que cada Estado federal es el responsable principal en el ámbito de la educación y la cultura, y que carece de una biblioteca nacional "clásica". Más bien se añadieron otras nuevas. Por un lado, la nueva tecnología permite modos más eficientes de cooperación, pues el networking es literalmente parte del tema general "trabajo global en red"; por otro, cada vez está más claro que los enfoques centralizados o incluso centralistas, a la vista de la general escasez de recursos, constituyen un éxito considerable. Además, el usuario, gracias a las nuevas posibilidades aparentemente ilimitadas de la búsqueda de información en la Red, se ha emancipado de las bibliotecas y de los servicios de información que les proporcionan y se ha hecho más exigente. Espera un servicio "todo en uno" lo más amplio posible; es decir, le gustaría que, como en el supermercado, se pudiera encontrar todo bajo el mismo techo.

Por eso, la cooperación no puede detenerse en el reparto del trabajo entre bibliotecas. Hoy es necesario que cooperen intensivamente $\mathrm{y}$ de modo interdisciplinar sobre todo con otras instituciones del mundo del saber, como archivos y museos, así como con el contexto científico. De esta cooperación interdisciplinar o mixta resultarán nuevos servicios y una mayor calidad de los mismos. Esto significa que el servicio total de la biblioteca no se orienta ya a los medios disponibles, sino que, consecuentemente, debe ser pensado y concebido desde el punto de vista del usuario, de sus expectativas y necesidades. En la era digital adquiere especial significado el aspecto de visibilidad y acceso a los servicios. Esto queda bien claro en el nuevo servicio cooperativo (digital) de las bibliotecas alemanas que a continuación presentaré brevemente: las llamadas bibliotecas virtuales.

\subsection{Bibliotecas virtuales especia- lizadas}

A partir del programa de las colecciones nacionales especiales al que antes me he referido, la $D F G$ impulsa desde hace unos años la creación de las llamadas bibliotecas virtuales especializadas ( $\mathrm{ViFa}$ ) o portales temáticos, que son entendidas en líneas generales como:

- almacenamiento de todos los recursos de información temáticamente relevantes;

- todos, digitales y convencionales;

- bajo una única interfaz de búsqueda;

- posibilidad de una metabúsqueda en los recursos de información más importantes;

- comprobación inmediata de la disponibilidad del documento;
- subsiguiente prestación del servicio de suministro del documento;

Surgen por regla general a partir de la cooperación entre las bibliotecas del programa de colecciones temáticas especiales o entre bibliotecas especializadas, que cuentan con participación de científicos $\mathrm{y}$ de sociedades científicas.

La $D F G$ prevé para las ViFa patrocinadas por ella unas determinadas características en la disposición y configuración de portales y de sus funcionalidades, es decir, una serie de requisitos mínimos. En concreto, son los siguientes:

- servicio de nuevas adquisiciones (para monografías);

- servicio de sumarios de revistas;

- metabúsqueda en las principales bases de datos especializadas;

- selección de las principales y más relevantes fuentes especializadas de internet;

- incorporación de nuevos re-

\section{Ejemplo de una cooperación interdisciplinar en un portal especializado: Ia Biblioteca Federal Bávara Online}

Desde 2002, un grupo de bibliotecas bávaras bajo la dirección de la BSB y en estrecha colaboración con otras instituciones culturales y de otro tipo, como la Oficina Federal Bávara de Estadística y la Oficina Federal Bávara de Topografía, ofrece un portal especializado de carácter cultural sobre Baviera. El usuario interesado puede encontrar las informaciones y materiales más variados sobre todas las cuestiones relativas a la historia y la cultura de la región. Comprende desde consultas en la bibliografía bávara, a documentos a texto completo, como por ejemplo actas de las sesiones parlamentarias, mapas históricos y topográficos, fotos, gráficos, así como películas y documentos sonoros. Es un servicio multimedia en el sentido más propio del término, además de cómodo, pues dispone de una búsqueda centralizada de personas y lugares, así como de temas y acontecimientos.

http://www. bayerische-landesbibliothek-online.de/? 
cursos de información en formato digital.

Como ejemplo, se puede consultar la ViFa de historia, llamada Chronicon que ha desarrollado mi propia biblioteca en los últimos dos años.

http://www.bsb-muenchen.del index.php?id $=148 \& L=\&$ styl

Todos los portales especializados o ViFa están a su vez -con independencia de la especialidad- incluidos en Vascoda, una iniciativa para la mejora del suministro de información especializada que cubre todo el ámbito alemán, algo así como un "supraportal" de todos los portales especializados.

\section{http://www.vascoda.de}

\subsection{Consorcios y licencias nacio- nales}

Una buena parte de los recursos digitales que ofrecen los $\mathrm{ViFa}$ son de carácter comercial, es decir, la biblioteca o el sistema bibliotecario, en cada caso, debe ocuparse de gestionar las correspondientes licencias. En Alemania éstas no se adquieren por lo general individualmente, sino, como ocurre también en España, de modo consorciado y de forma cooperativa. En Alemania dominan los llamados consorcios regionales, que no siempre coinciden con las agrupaciones regionales de bibliotecas, a las que me referí anteriormente, de los que en la actualidad existen 10. A ellos hay que añadir los de las diferentes instituciones de investigación, como por ejemplo los Institutos Max-Planck. Junto con los consorcios nacionales de Austria y de la Suiza alemana, los alemanes constituyen un grupo de trabajo, la German-Austrian-Switzerland-Consortia-Organisation, denominada Gasco. Con ello, la cooperación bibliotecaria regional y nacional ha pasado a ser internacional.

\section{http://www.gasco.de}

El factor cooperativo a nivel nacional se ha reforzado desde hace dos años en el ámbito de los consorcios debido a que la $D F G$, en el seno del ya mencionado plan de colecciones temáticas especiales, permite adquirir licencias nacionales a través de Gasco para determinadas bases de datos especializadas, revistas electrónicas y e-books, que son escasamente solicitados a nivel local. En estos casos se adquiere una licencia nacional para todo el país a un precio global. Así, en Alemania todos los usuarios (no comerciales) interesados en esta oferta tienen la posibilidad de acceder online, desde su propia mesa de trabajo o desde la biblioteca que les resulte más próxima, a los recursos de información contratados mediante esas licencias, siempre que se hayan registrado como usuarios.

\subsection{Archivo digital de larga du- ración}

La estructura federal del país, con las agrupaciones regionales de bibliotecas y la Biblioteca Nacional Virtual, tiene como consecuencia que en Alemania, para obras impresas sin necesidad de acuerdo expreso, exista de hecho un concepto compartido o cooperativo de biblioteca de depósito. En Baviera se da incluso el concepto de una biblioteca regional de depósito (lo que constituye una excepción en Alemania).

De modo análogo se procede en el mundo digital. La complejidad de las cuestiones del archivo digital de larga duración se ha afrontado en Alemania desde el principio, intencionadamente, desde una perspectiva cooperativa y participativa. Desde 2002 existe un organismo competente en las cuestiones del archivo digital de larga conservación, denominado Nestor. Es una iniciativa interdisciplinar, como es habitual en el mundo digital, en la que participan también archivos (el Archivo Federal Central y los archivos de los respectivos Estados federales) y al que en los últimos tiempos se han asociado también los museos. En la actualidad, hay varios proyectos en los que se están probando, tanto desde un punto de vista organizativo como técnico, los procesos idóneos para el archivo masivo de documentos. En este asunto existe una estrecha cooperación con proyectos semejantes en otros países, especialmente una participación activa en los intentos de lograr un proyecto conjunto europeo, como por ejemplo la Alliance for Preservation.

En Baviera, la $B S B$ ha comenzado hace dos años, con el apoyo financiero de la $D F G$, un proyecto que se encuentra ya en fase de desarrollo. Junto con la otra institución participante, el Centro de Cálculo Leibniz de Munich, uno de los grandes centros de cálculo de Europa, se han alojado y archivado, una vez provistas de los correspondientes metadatos, publicaciones que originalmente están en formato electrónico en un workflow apoyado informáticamente y constituido por los productores, por ejemplo editoriales, así como por instituciones públicas que también publican, como la Oficina Federal Bávara de Estadística. El usuario tiene así acceso por medio del catálogo colectivo a la copia digital del archivo. El sistema se llama BABS (Sistema Bibliotecario de Archivo y Acceso) y en los próximos años va a experimentar un considerable desarrollo. Está previsto que con la digitalización masiva que comenzará en 2008 en colaboración con Google y la incorporación progresiva de documentos originales digitales, cada año se incremente en un volumen de 100 terabytes aproximadamente.

\section{Conclusión}

La cooperación entre bibliotecas y de ellas con otras instituciones, es una obligación en la era digital. Pero la cooperación tiene, naturalmente, un precio: 
- Deben tenerse en cuenta los costes inmediatos de los trabajos de coordinación, que pueden ser muy diferentes para los diversos participantes.

- Asimismo, no hay que perder de vista que según las circunstancias los participantes pueden beneficiarse de muy diferente forma y que sólo podrán imponer sus intereses con condiciones. Lema: la cooperación es por lo general un compromiso.

- Una iniciativa de cooperación puede suponer, en casos extremos, una amenaza de la propia identidad o incluso puede llevar a perderla y a hacer peligrar su existencia.

Las circunstancias concretas determinarán que una cooperación implique ganancias para quienes participan en ella. Estoy convencido, en todo caso, de que pese a todos los problemas en el futuro hemos de cooperar todavía más y que, en consecuencia, nos encontramos al comienzo de una "era cooperativa".

\section{Notas}

1. Texto de la conferencia inaugural de la $X V$ Asamblea anual de Rebiun (Red de Bibliotecas
Universitarias), pronunciada en La Coruña el 21 de noviembre de 2007. Agradezco a mi colega y amigo Víctor Sanz su ayuda en la traducción al español del texto.

2. Para situar el contexto, proporciono muy sumariamente algunos datos de carácter general sobre Baviera: es el Estado federal más grande de Alemania en cuanto a la extensión $(70.000$ $\mathrm{km}^{2}$ ) y el segundo en cuanto a la población (más de doce millones de habitantes). En el ámbito bibliotecario existe una estrecha cooperación entre la Biblioteca Estatal de Baviera, las 10 bibliotecas universitarias, las 17 bibliotecas de otros centros de educación superior y las 9 públicas regionales.

Klaus Kempf, Biblioteca Estatal de Baviera, Alemania.

Klaus.Kempf@bsb-muenchen.de

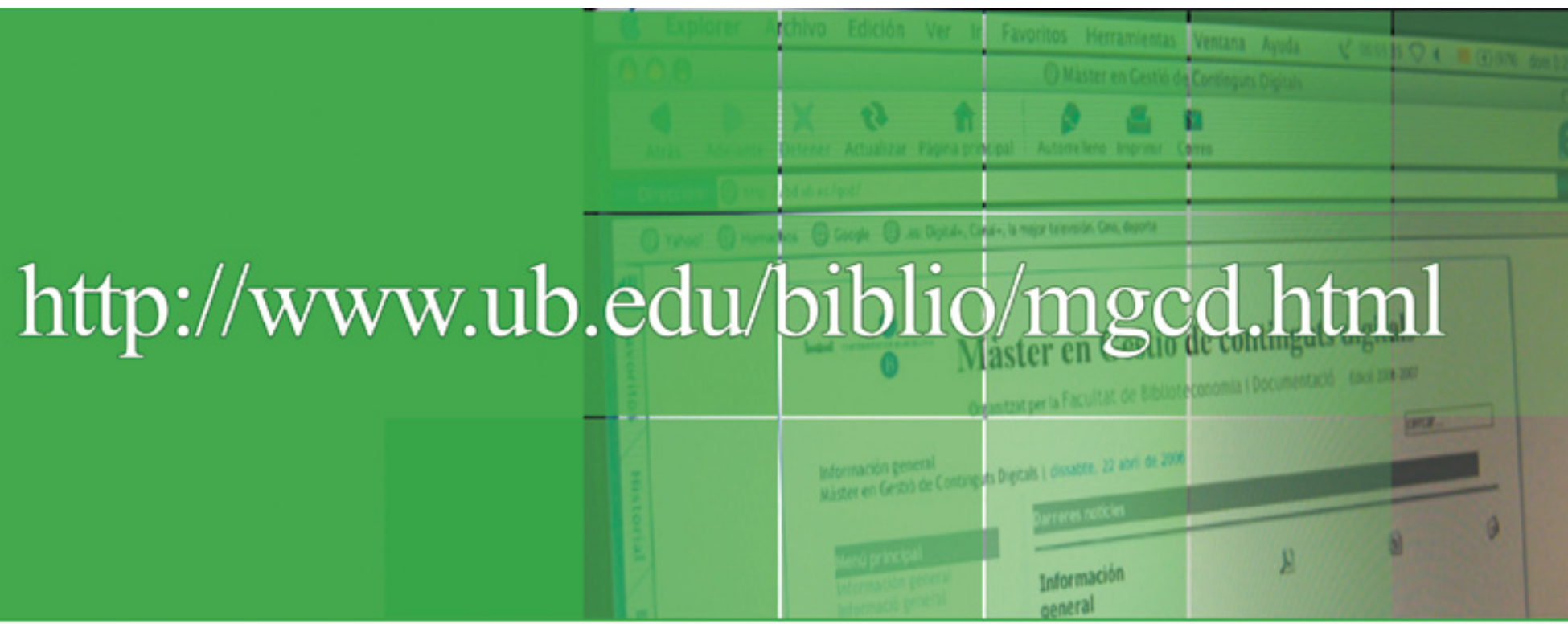

\section{Máster en Gestión de Contenidos Digitales}

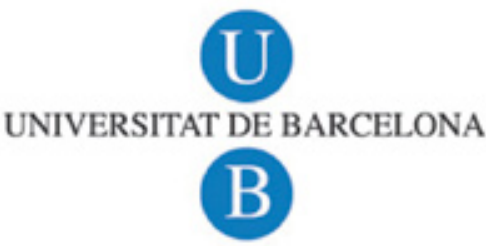

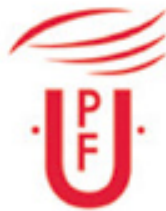

U NIVERSITAT

POMPEU FABRA 